\title{
Modeling of a two-phase swirling turbulent flow in the separation chamber of the centrifugal apparatus
}

\author{
Nikolay Evseev a and Alexander Shvab \\ Tomsk State, University, Russia
}

\begin{abstract}
In this paper a two-phase (gas - solid particles) swirling turbulent flow in the separation chamber of a centrifugal apparatus is considered. The results of mathematical modeling of flow at different settings are shown.
\end{abstract}

This paper presents a two-phase (gas - solids) swirling turbulent flow in the separation chamber of the centrifugal apparatus with an additional supply of gas (Fig. 1). Through the section A-A the gas flow is supplied with particles having radial and tangential velocity components. Through the section B-B an additional supply of gas without solid is provided. The flow in the vortex chamber is axisymmetric by its nature.

Through the section $\mathrm{C}-\mathrm{C}$ the swirling flow together with the gas fraction of fine particles leaves the working area of the vortex chamber. Through the section B-B the incoming gas stream filters larger particles of the particulate fraction, returning finer particles to the working zone of the classifier. Large particles, due to the predominance of the centrifugal force over the aerodynamic force, leave the vortex chamber through the cross section B-B. All solid walls of the apparatus can rotate about the axis $\mathrm{O}-\mathrm{Z}$, giving additional rotation of the gas flow with the solid phase and thereby aligning the field of circumferential velocity vector components. To describe the swirling gas flow in the separation chamber a set of the Reynolds equations is used. For its closure the generalized Boussinesq model is applied according to which the Reynolds stresses are considered to be proportional to the mean flow strain rate and the coefficient of eddy viscosity $v$ t. Thus, the system of Reynolds equations in the cylindrical coordinate system in the conservative dimensionless form for an incompressible viscous fluid taking into account the axial symmetry $(\partial / \partial \varphi=0)$ can be written as follows:

$$
\begin{gathered}
\frac{\partial}{\partial r}\left[\left(u_{r} r \varepsilon\right)\right]+\frac{\partial}{\partial z}\left[\left(u_{z} r \varepsilon\right)\right]=0 ; \quad \varepsilon+\sum \varepsilon_{j}=1 ; \\
\frac{\partial r u_{r}}{\partial \tau}+\frac{\partial}{\partial r}\left(r u_{r}^{2}\right)+\frac{\partial}{\partial z}\left(r u_{z} u_{r}\right)-\frac{1}{\operatorname{Re}}\left\{\frac{\partial}{\partial r}\left[r\left(1+v_{t}\right) \frac{\partial u_{r}}{\partial r}\right]+\frac{\partial}{\partial z}\left[r\left(1+v_{t}\right) \frac{\partial u_{r}}{\partial z}\right]\right\}= \\
=u_{\varphi}^{2}-r \frac{\partial p}{\partial r}+\frac{r}{\operatorname{Re}}\left[\frac{\partial v_{t}}{\partial r} \frac{\partial u_{r}}{\partial r}+\frac{\partial v_{t}}{\partial z} \frac{\partial u_{z}}{\partial r}-\frac{u_{r}}{r^{2}}\left(1+v_{t}\right)\right]-\frac{1}{\varepsilon \rho} \sum_{j=1}^{N} \varepsilon_{j} \rho_{j} \frac{\left(u_{r}-w_{r j}\right)}{S t k} \zeta
\end{gathered}
$$

\footnotetext{
${ }^{a}$ Corresponding author: nikolay90@sibmail.com
}

This is an Open Access article distributed under the terms of the Creative Commons Attribution License 4.0, which permits unrestricted use, distribution, and reproduction in any medium, provided the original work is properly cited. 


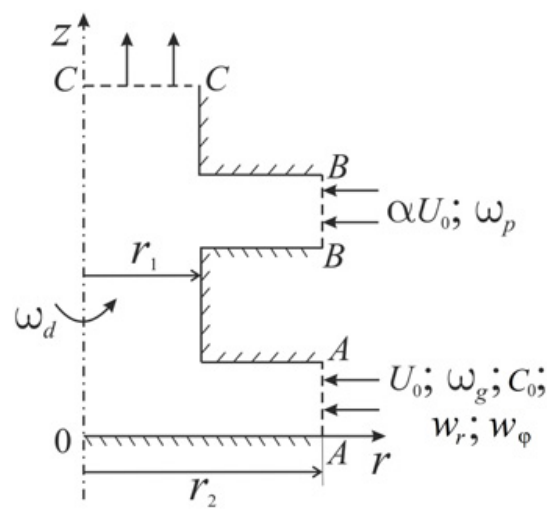

Figure 1. Scheme of the separation zone of the vortex chamber.
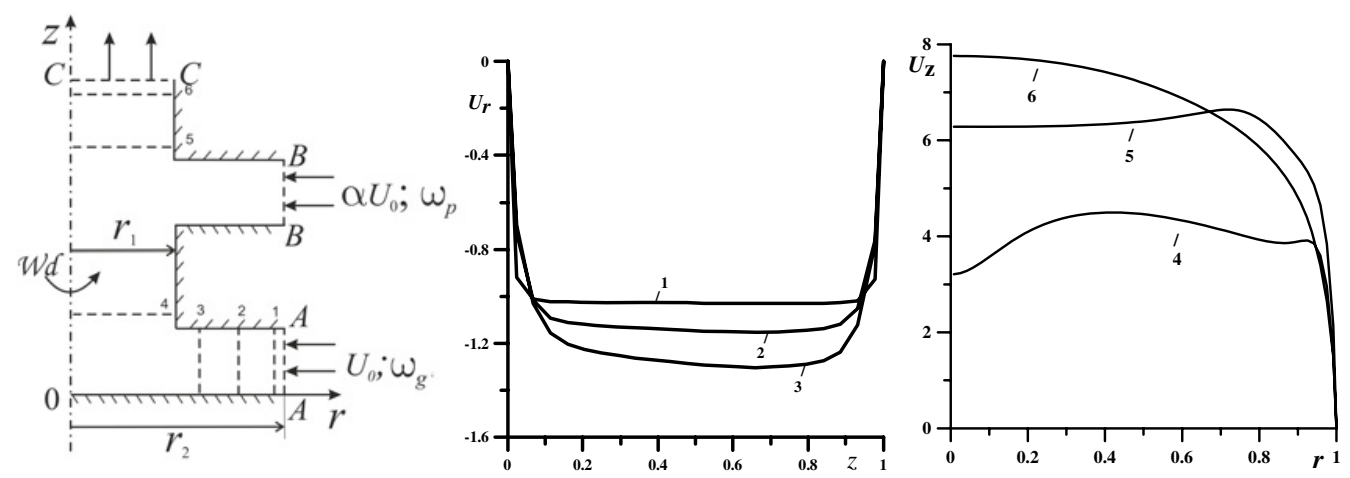

Figure 2. Velocity profile $u_{r}, u_{z}$ in different sections.

$$
\begin{gathered}
\frac{\partial r u_{z}}{\partial \tau}+\frac{\partial}{\partial z}\left(r u_{z}^{2}\right)+\frac{\partial}{\partial r}\left(r u_{z} u_{r}\right)-\frac{1}{\operatorname{Re}}\left\{\frac{\partial}{\partial r}\left[r\left(1+v_{t}\right) \frac{\partial u_{z}}{\partial r}\right]+\frac{\partial}{\partial z}\left[r\left(1+v_{t}\right) \frac{\partial u_{z}}{\partial z}\right]\right\}= \\
=-r \frac{\partial p}{\partial z}+\frac{r}{\operatorname{Re}}\left[\frac{\partial v_{t}}{\partial r} \frac{\partial u_{r}}{\partial z}+\frac{\partial v_{t}}{\partial z} \frac{\partial u_{z}}{\partial z}\right]-\frac{1}{\varepsilon \rho} \sum_{j=1}^{N} \varepsilon_{j} \rho_{j} \frac{\left(u_{z}-w_{z j}\right)}{S t k} \zeta \\
\frac{\partial r u_{\varphi}}{\partial \tau}+\frac{\partial}{\partial r}\left(r u_{r} u_{\varphi}\right)+\frac{\partial}{\partial z}\left(r u_{z} u_{\varphi}\right)-\frac{1}{\operatorname{Re}}\left\{\frac{\partial}{\partial r}\left[r\left(1+v_{t}\right) \frac{\partial u_{\varphi}}{\partial r}\right]+\frac{\partial}{\partial z}\left[r\left(1+v_{t}\right) \frac{\partial u_{\varphi}}{\partial z}\right]\right\}= \\
=-u_{r} u_{\varphi}-\frac{1}{\operatorname{Re}}\left[\left(1+v_{t}\right) \frac{u_{\varphi}}{r}+u_{\varphi} \frac{\partial v_{t}}{\partial r}\right]-\frac{1}{\varepsilon \rho} \sum_{j=1}^{N} \varepsilon_{j} \rho_{j} \frac{\left(u_{\varphi}-w_{\varphi j}\right)}{S t k} \zeta .
\end{gathered}
$$

There are different approaches to the modeling of turbulent viscosity. In this paper, a differential $\ll \mathrm{k}-\omega »$ Wilcox turbulence model [1] is used. According to this model of turbulence two equations 

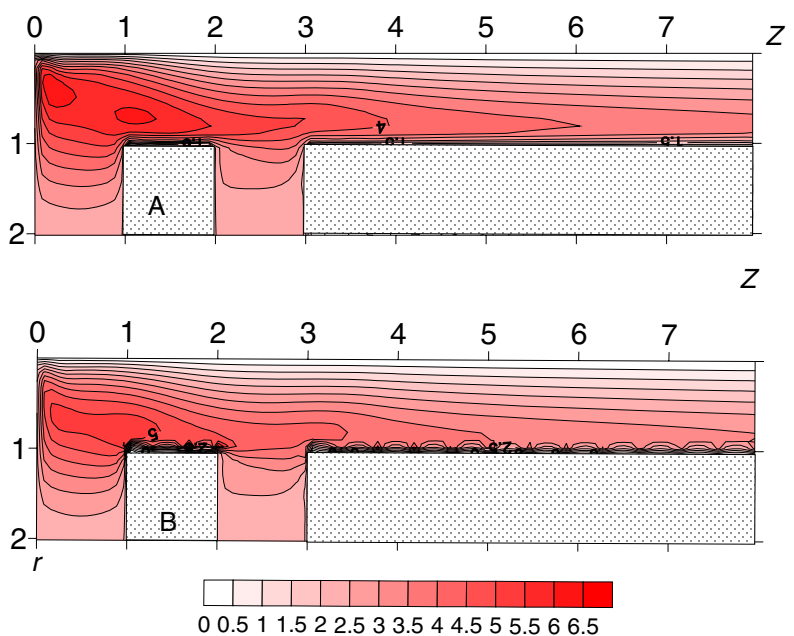

Figure 3. Field distributions of $u_{\varphi}, w_{\varphi}$.

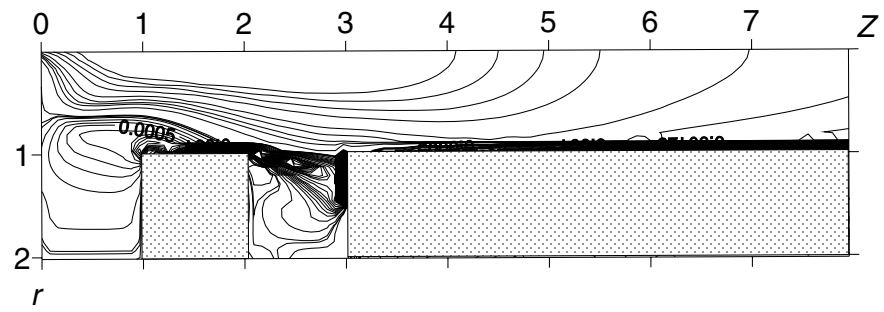

Figure 4. Distribution of contours of the solid phase volume concentration.

are added for the transfer of kinetic energy of turbulent fluctuations $\mathrm{k}$ and the specific dissipation rate of kinetic energy $\omega$. In the cylindrical coordinate system, these equations take the following form:

$$
\begin{gathered}
\frac{\partial r k}{\partial \tau}+\frac{\partial}{\partial r}\left(r u_{r} k\right)+\frac{\partial}{\partial z}\left(r u_{z} k\right)=\frac{1}{\operatorname{Re}}\left\{\frac{\partial}{\partial r}\left[r\left(1+v_{t} \sigma^{*}\right) \frac{\partial k}{\partial r}\right]+\frac{\partial}{\partial z}\left[r\left(1+v_{t} \sigma^{*}\right) \frac{\partial k}{\partial z}\right]\right\}+G-\beta^{*} r k \omega \\
\frac{\partial r \omega}{\partial \tau}+\frac{\partial}{\partial r}\left(r u_{r} \omega\right)+\frac{\partial}{\partial z}\left(r u_{z} \omega\right)=\frac{1}{\operatorname{Re}}\left\{\frac{\partial}{\partial r}\left[r\left(1+v_{t} \sigma\right) \frac{\partial \omega}{\partial r}\right]+\frac{\partial}{\partial z}\left[r\left(1+v_{t} \sigma\right) \frac{\partial \omega}{\partial z}\right]\right\}+\gamma G \frac{\omega}{k}-\beta r \omega^{2} \\
G=\frac{v_{t} r}{\operatorname{Re}}\left\{\left(\frac{\partial u_{\varphi}}{\partial r}-\frac{u_{\varphi}}{r}\right)^{2}+\left(\frac{\partial u_{r}}{\partial z}+\frac{\partial u_{z}}{\partial r}\right)^{2}+\left(\frac{\partial u_{\phi}}{\partial z}\right)+2\left[\left(\frac{\partial u_{r}}{\partial r}\right)^{2}+\left(\frac{u_{r}}{r}\right)^{2}+\left(\frac{\partial u_{z}}{\partial z}\right)^{2}\right]\right\} \\
v_{t}=\operatorname{Re} \frac{k}{\omega} .
\end{gathered}
$$


The values of the constants used in the turbulence model [1] are: $\beta=3 / 40, \beta^{*}=9 / 100, \gamma=5 / 9$, $\sigma=1 / 2, \sigma^{*}=1 / 2$. The equations of motion of the solid phase are of the form:

$$
\begin{gathered}
\frac{\partial r w_{r j}}{\partial \tau}+\frac{\partial}{\partial r}\left(r w_{r j}^{2}\right)+\frac{\partial}{\partial z}\left(r w_{z j} w_{r j}\right)-w_{\varphi j}^{2}=\frac{\left(u_{r}-w_{r j}\right)}{S t k} r \zeta_{j}+\Phi_{r j} ; \\
\frac{\partial r w_{\varphi j}}{\partial \tau}+\frac{\partial}{\partial r}\left(r w_{r j} w_{\varphi j}\right)+\frac{\partial}{\partial z}\left(r w_{z j} w_{\varphi j}\right)+w_{r j} w_{\varphi j}=\frac{\left(u_{\varphi}-w_{\varphi j}\right)}{S t k} r \zeta_{j}+\Phi_{\varphi j} ; \\
\frac{\partial r w_{z j}}{\partial \tau}+\frac{\partial}{\partial r}\left(r w_{r j} w_{z j}\right)+\frac{\partial}{\partial z}\left(r w_{z j}^{2}\right)=\frac{\left(u_{z}-w_{z j}\right)}{S t k} r \zeta_{j}+\frac{r}{F r}+\Phi_{z j} .
\end{gathered}
$$

The transport equation of the solid phase volume concentration $\varepsilon \mathrm{j}$ :

$$
\frac{\partial r \varepsilon_{j}}{\partial \tau}+\frac{\partial r \varepsilon_{j} w_{r}}{\partial r}+\frac{\partial r \varepsilon_{j} w_{z}}{\partial z}=\frac{\partial}{\partial r}\left[\frac{r}{\operatorname{Re}}\left(\frac{1}{S c}+\frac{v_{t}}{S c_{t}}\right) \frac{\partial \varepsilon_{j}}{\partial r}\right]+\frac{\partial}{\partial z}\left[\frac{r}{\operatorname{Re}}\left(\frac{1}{S c}+\frac{v_{t}}{S c_{t}}\right) \frac{\partial \varepsilon_{j}}{\partial z}\right] .
$$

Here $\varepsilon j$ - the volumetric solid phase concentration of the $j$-th fraction, Sc, Sct - the molecular and turbulent Schmidt numbers, respectively, and $\zeta \mathrm{j}=1+0.197 \cdot$ Rej0.63 $+2.6 \cdot 10-4 \cdot$ Rej1.38, where Rej - the Reynolds number, based on the particle size of the j-th fraction and the relative velocity of the particles and gas, Fr - the Froude number. The dimensionless form for a system of Eqs. ((1)-(3)) and (4) is obtained using the following scales: the averaged flow rate U0 is chosen as the characteristic velocity; the output channel radius $\mathrm{r} 1$ - as the characteristic length. For pressure and turbulent viscosity to be dimensionless additionally the characteristic density and kinematic viscosity of the gas are used. Solution of The system of transport equations of momentum and continuity equations is solved in variables "velocity - pressure" by the method of splitting velocity and pressure fields on the staggered finite-difference grid using the exponential scheme. Values of functions $\Phi$ rj, $\Phi z \mathrm{zj}, \Phi \varphi \mathrm{j}$ for the solid phase represent the artificial viscosity and its calculation is similar to that of the gas phase. At the entrance of the separation element (cross-section A-A, Fig. 1) the distributions of volume concentration, radial and circumferential velocity vector components for gas and solid phases, kinetic energy and specific dissipation rate were given. In the section B-B similar distributions were set only for the gas phase and the solid phase in the same section was specified by the Neumann conditions.

On the walls of the gas phase the slip condition was used, and in the output section C-C the Neumann condition was imposed. For the solid phase on the walls the partial slip conditions were set for the tangential velocity component, and for the normal velocity component - equal to zero. On the axis the symmetry condition was used. Figure 2 shows the distribution of the radial and axial components of the velocity vector in the working area in the cross sections.

Figure 3 shows the distribution of circumferential velocity vector components for a) gas $b$ ) particles with parameters: $\operatorname{Re}=5000, \alpha=0.1, \operatorname{Rg}=\mathrm{Rd}=\mathrm{Rp}=1$.

Figure 4 shows the distribution of the volume concentration of the solid phase with the parameters: $\operatorname{Re}=5000, \alpha=0.1, \operatorname{Rg}=\operatorname{Rd}=\operatorname{Rp}=1, \delta=15 \mathrm{mkm}$. This graph illustrates the process of separation of solid particles into small and large fractions.

This work was financially supported by the RFBR grant No. 13-08-00367-A.

\section{Reference}

[1] Wilcox D.C. Reassessment of the scale-determining equation for advanced turbulence models. // AIAA J. V. 26. No 11. P. 1299-1310 (1988) 